\title{
ON DOUBLE TRIGONOMETRIC INTEGRALS
}

\author{
BY \\ LEONARD D. BERKOVITZ(1)
}

In the one-dimensional case Wolf [3] and Zygmund [5] $\left({ }^{2}\right)$ have shown that given a trigonometric integral $\int_{-\infty}^{+\infty} e^{i x u} d \phi(u)$ where $\phi(u)$ is of bounded variation on every finite interval and behaves with a certain regularity as $|u| \rightarrow \infty$, then a trigonometric series $\sum_{m=-\infty}^{+\infty} a_{m} e^{i m x}$ can be found such that on any interval $0<\epsilon \leqq x \leqq 2 \pi-\epsilon$ the difference.

$$
\Delta_{\omega}(x)=\int_{-\omega}^{\omega} e^{i x u} d \phi(u)-\sum_{m=-[\omega]}^{[\omega]} a_{m} e^{i m x}
$$

converges uniformly (or more generally is uniformly summable $(C, k)$ ) to zero as $\omega \rightarrow \infty$. Moreover, the coefficients $a_{m}$ exhibit a regularity in their behavior as $|m| \rightarrow \infty$ which is connected with that of $\phi(u)$. This result enables one to obtain theorems about the behavior of trigonometric integrals from the known theorems about trigonometric series. It is also shown in [5] that integrals of the type $\int_{-\infty}^{+\infty} e^{i x u} d \phi(u)$ are equiconvergent with integrals of the form $\int_{-\infty}^{+\infty} e^{i x u} \alpha(u) d u$, where $\alpha(u)$ is continuous. The purpose of this paper is to extend these results in certain cases to double trigonometric integrals, using restricted and circular convergence. When circular convergence is used extensions to higher dimensions are also possible.

1.1. Definitions and notations. Let $\phi$ be a real-valued set function defined on the class of Borel sets $\mathfrak{B}$ in the plane and satisfying the conditions

$$
\begin{aligned}
& \text { (a) }|\phi(S)|<\infty \text { if } S \text { is bounded, } \\
& \text { (b) } \phi\left(\bigcup_{i=1}^{\infty} S_{i}\right)=\sum_{i=1}^{\infty} \phi\left(S_{i}\right)
\end{aligned}
$$

whenever $S_{i} \wedge S_{j}=0$ for $i \neq j$ and $\bigcup_{i=1}^{\infty} S_{i}$ is bounded. If $\phi$ is also non-negative, then we shall call it a measure. It is well known (see [2]) that under these assumptions the functions of set $\phi^{+}(S), \phi^{-}(S)$, and $W(\phi ; S)$ defined by

$$
\begin{aligned}
\phi^{+}(S) & =\sup \{\phi(E) ; E \subset S, E, S \in \mathfrak{B}\}, \\
\phi^{-}(S) & =-\inf \{\phi(E) ; E \subset S, E, S \in \mathfrak{B}\}, \\
W(\phi ; S) & =\phi^{+}(S)+\phi^{-}(S)
\end{aligned}
$$

Presented to the Society, December 1, 1951; received by the editors December 10, 1951.

(1) The research for this paper was carried out while the author was a United States Atomic Energy Commission Pre-Doctoral Fellow at the University of Chicago.

(2) Numbers in brackets refer to the list of references at the end of the paper. 
are measures and satisfy the relations

$$
\begin{aligned}
\text { (a) } \phi(S) & =\phi^{+}(S)-\phi^{-}(S), \\
\text { (b) } W(\phi ; S) & =\sup \left\{\sum_{i=1}^{n}\left|\phi\left(E_{i}\right)\right|\right\}
\end{aligned}
$$

where in (b) the supremum is taken over all finite disjoint sums of Borel sets $E_{i}$ contained in $S$. The function $\phi^{+}$is called the upper variation of $\phi$ on $S$, $\phi^{-}$the lower variation, and $W(\phi ; S)$ the total variation of $\phi$ on $S$. If $\phi$ is a complex-valued set function defined on the class $\mathfrak{B}$, say, $\phi(S)=\phi_{1}(S)+i \phi_{2}(S)$ with $\phi_{1}$ and $\phi_{2}$ real-valued, and if $\phi$ satisfies the conditions 1 (a) and 1(b), then $\phi_{1}$ and $\phi_{2}$ will also satisfy these conditions. Thus in view of 2(a) we can write

$$
\phi=\phi_{1}^{+}-\overline{\phi_{1}^{-}}+i\left(\phi_{2}^{+}-\overline{\phi_{2}}\right)
$$

where $\phi_{1}^{+}, \phi_{1}^{-}, \phi_{2}^{+}$, and $\phi_{2}^{-}$are measures on the class of Borel sets in the plane. Furthermore, for complex-valued functions $\phi, W(\phi ; S)$ is defined as the sum of the real and imaginary total variations. Thus $W(\phi ; S)=W\left(\phi_{1} ; S\right)+$ $W\left(\phi_{2} ; S\right)$. Given any such $\phi$, a bounded Borel set $A$, and a Borel measurable function $f\left(u_{1}, u_{2}\right)$, we are thus led to define

$$
\iint_{A} f\left(u_{1}, u_{2}\right) d \phi=\iint_{A} f d \phi_{1}^{+}-\iint_{A} f d \overline{\phi_{1}}+i \iint_{A} f d \phi_{2}^{+}-i \iint_{A} f d \overline{\phi_{2}},
$$

provided each integral on the right exists and is finite. If any one of the integrals on the right does not exist or is infinite, then we shall say that $\iint_{A} f d \phi$ does not exist. As is customary, we denote the integrals with respect to the measure $W(\phi ; S)$ by $\iint_{A} f|d \phi|$. Thus, $W(\phi ; A)=\iint_{A}|d \phi|$.

So far we have defined the integral $\iint_{A} f d \phi$ only for bounded Borel sets. If $A$ is unbounded and

$$
\lim _{R \rightarrow \infty} \iint_{A \wedge C_{R}}|f||d \phi|<\infty,
$$

where $C_{R}$ is a closed circle with center at origin and radius $R$, we say that $f$ is integrable over $A$ and set

$$
\iint_{A} f d \phi=\lim _{R \rightarrow \infty} \iint_{A \wedge C_{R}} f d \phi
$$

Whenever $A$ is the entire plane $E_{2}$ and $f$ is integrable over $E_{2}$, we write $\iint_{E_{2}} f d \phi$. It may happen that, although $f$ is not integrable on $E_{2}$, the integrals of $f$ taken over finite rectangles $Q$ or circles $K$ tend to a limit as the rectangles or circles fill up the plane. This situation will be described by saying that the integrals over $Q$ or $K$, as the case may be, converge in a certain sense to a 
limit. More precisely, if $Q$ is a closed rectangle with center at the origin and with sides of length $2 S$ by $2 T$, we write

$$
\iint_{Q} f d \phi=\int_{-S}^{S} \int_{-T}^{T} f d \phi
$$

and say that the integral

$$
\int_{-\infty}^{+\infty} \int_{-\infty}^{+\infty} f d \phi
$$

converges to a limit $l$ in the Pringsheim sense if for arbitrary $\epsilon>0$ there exist positive real numbers $S_{0}$ and $T_{0}$ such that, whenever $S>S_{0}$ and $T>T_{0}$, the inequality $\left|\int_{-S}^{S} \int_{-T}^{T} f d \phi-l\right|<\epsilon$ holds. We shall not make any use of Pringsheim convergence, but shall be concerned with restricted convergence and circular convergence. The integral (5) is said to converge restrictedly to a limit $l$ if for any positive real constants $A$ and $B$ it is true that for every $\epsilon>0$ positive real numbers $S_{0}$ and $T_{0}$ can be found with the property that, whenever $S>S_{0}, T>T_{0}$, and $A \leqq S / T \leqq B$, the inequality $\left|\int_{-S}^{S} \int_{-T}^{T} f d \phi-l\right|<\epsilon$ holds. The integral (5) is said to converge circularly to a limit $l$ in case given any $\epsilon>0$ there is an $R_{0}>0$ such that for $R>R_{0}$ we have $\left|\iint_{C_{R}} f d \phi-l\right|$ $<\epsilon$, where $C_{R}$ is the closed circle with center at the origin and radius $R$. Implicit in the above discussion is the convention that by $\int_{-\infty}^{+\infty} \int_{-\infty}^{+\infty} f d \phi$ we mean the Cauchy limit taken in the appropriate sense, while by $\iint_{E_{2}} f d \phi$ we mean the Lebesgue-Stieltjes integral of $f$ over the plane. This notation will be more or less adhered to throughout the paper. Clearly, if $\iint_{E_{2}} f d \phi$ exists, so does $\int_{-\infty}^{+\infty} \int_{-\infty}^{+\infty} f d \phi$ and the two are equal, regardless of the sense in which the Cauchy limit is taken. Hence in such situations a notational distinction is not really needed, and it is to this fact that the phrase "more or less" refers.

Before proceeding to the discussion of trigonometric integrals we list some conventions and notations which will be used throughout the paper. Unless otherwise stated, all sets to be henceforth considered will be Borel sets, all set functions will be complex-valued and have as their domain the class $\mathfrak{B}$, and the term "measurable" will be taken to mean "Borel measurable." Lower case italic letters with no subscripts attached will generally indicate points, while letters with subscripts will indicate coordinates. Thus, by $u \equiv\left(u_{1}, u_{2}\right)$ we mean the point $u$ with coordinates $u_{1}$ and $u_{2}$. The expression $i\left(x_{1} u_{1}+x_{2} u_{2}\right)$ will be denoted by $i x u$ and the norm of $u$ will be denoted by $|u|$; i.e. $|u|=\left(u_{1}^{2}+u_{2}^{2}\right)^{1 / 2}$. This will not cause confusion with the ordinary use of the absolute value sign since whenever we wish to speak of the absolute value of a coordinate we write $\left|u_{i}\right|$. To indicate that we are integrating a function $f(u, v)$ with respect to $u$, say, we write $\iint_{A} f(u, v) d \phi(u)$; if there is only one variable point we write $\iint_{A} f(u) d \phi$ or $\iint_{A} f d \phi$. In this connection we agree that when we say that a function of set is absolutely continuous we mean absolutely continuous with respect to Lebesgue measure. The closed 
circle with center at the origin and radius $R$ shall be denoted by $C_{R}$. Following Saks, we define

$$
A \ominus B=[(A-B) \circ]^{-}
$$

where the symbol $A^{\circ}$ stands for the interior of $A$ and $A^{-}$is the closure of $A$. Finally, we let $(u ; A)$ stand for the translate of the set $A$ by the vector $u$; i.e. $(u ; A)=E[u+a, a \in A]$.

1.2. Formal products of trigonometric integrals. A double trigonometric integral is an integral of the form

$$
\int_{-\infty}^{+\infty} \int_{-\infty}^{+\infty} e^{i x u} d \phi(u)
$$

Clearly, double trigonometric series are special cases of the integral (6). Given two integrals

$$
B=\int_{-\infty}^{+\infty} \int_{-\infty}^{+\infty} e^{i x u} d \phi(u) \text { and } G=\int_{-\infty}^{+\infty} \int_{-\infty}^{+\infty} e^{i x u} d \psi(u)
$$

we look at the case in which $B$ and $G$ reduce to series with sufficiently well behaved coefficients, examine the definitions of formal product (see [1], for example), and are led to propose the following definition for the product $P$ of $B$ and $G$. First form the point function $\phi(-u ; A)$, and then consider the function of $\operatorname{set} \mathrm{X}(A)=\iint_{E_{2}} \phi(-u ; A) d \psi(u)$. The product integral $P$ is then taken to be $\int_{-\infty}^{+\infty} \int_{-\infty}^{+\infty} e^{i x u} d \mathrm{X}(u)$. Of course, the above discussion is purely heuristic and leaves much to be verified. This, however, is not difficult, and the next two lemmas are devoted to this purpose.

Lemma 1. Let $\phi$ and $\psi$ be non-negative set functions satisfying conditions 1 (a) and 1(b). Then for each fixed bounded set $A$, the functions $\phi(u ; A)$ and $\psi(u ; A)$ are measurable functions of $u$. Moreover, if $\phi$ or $\psi$ is absolutely continuous, or the set $A$ is $a G_{\delta}$, then,

$$
\iint_{E_{2}} \phi(-u ; A) d \psi(u)=\iint_{E_{2}} \psi(-u ; A) d \phi(u)
$$

where the integrals may take on the value $+\infty$.

Except for very minor modifications the lemma and its proof are contained in the proof of Theorem 11.1 in Chapter III of Saks [2]. We omit the proof here.

Lemma 2. Let $\phi$ and $\psi$ be set functions satisfying conditions 1(a) and 1(b). Also for each fixed bounded set $A$ let there exist a constant $K_{A}$ such that $|\phi(-u ; A)| \leqq K_{A}$, and suppose that $\iint_{E_{2}}|d \psi|<\infty$. Then the set function $\mathrm{X}(A)$ given by 


$$
\mathrm{X}(A)=\iint_{E_{2}} \phi(-u ; A) d \psi
$$

is defined for all bounded sets $A$, satisfies conditions 1(a) and 1(b), and if either $\phi$ or $\psi$ is absolutely continuous, the equation

$$
\mathrm{X}(A)=\iint_{E_{2}} \phi(-u ; A) d \psi=\iint_{E_{2}} \psi(-u ; A) d \phi
$$

holds.

Decomposing $\phi$ as in (3) and then applying Lemma 1 immediately shows that $\phi(-u ; A)$ is measurable. With this fact established, the hypotheses that $|\phi(-u ; A)| \leqq K_{A}$ and $\iint_{E_{2}}|d \psi|<\infty$ say that the function $\mathrm{X}(A)$ is actually defined for all bounded sets and satisfies condition $1(\mathrm{a})$. To show that $\mathrm{X}(A)$ satisfies condition 1 (b) let $A_{0}=\bigcup_{i=1}^{\infty} A_{i}$, where $A_{0}$ is bounded and $A_{i} \wedge A_{j}=0$ if $i \neq j$. Then

$$
\mathrm{X}\left(A_{0}\right)=\iint_{E_{2}} \phi\left(-u ; A_{0}\right) d \psi=\iint_{E_{2}}\left[\sum_{i=1}^{\infty} \phi\left(-u ; A_{i}\right)\right] d \psi .
$$

But

$$
\left|\sum_{i=1}^{N} \phi\left(-u ; A_{i}\right)\right| \leqq W\left(\phi ;\left(-u ; A_{0}\right)\right) \leqq K_{A} .
$$

Thus we may interchange the order of integration and summation in (11) and get

$$
\mathrm{X}\left(A_{0}\right)=\sum_{i=1}^{\infty} \iint_{E_{2}} \phi\left(-u ; A_{i}\right) d \psi=\sum_{i=1}^{\infty} \mathrm{X}\left(A_{i}\right)
$$

which is precisely $1(\mathrm{~b})$. Relation (10) is proved by first breaking both $\phi$ and $\psi$ into real and imaginary parts and then decomposing each of these into positive and negative variations. Corresponding to this there is a breakup of the integral $\iint_{E_{2}} \phi(-u ; A) d \psi$ into sixteen integrals, each of which is finite by the previous part of this lemma, and by Lemma 1 satisfies (8). Recombining these integrals then gives (10).

We now return to the integrals $B$ and $G$ in (7) and suppose that $\phi$ and $\psi$ satisfy the conditions of Lemma 2 . The formal product of $B$ and $G$ is now defined to be the integral

$$
P=\int_{-\infty}^{+\infty} \int_{-\infty}^{+\infty} e^{i x u} d \mathrm{X}(u)
$$

where $\mathrm{X}(A)$ is the set function given by (9). Symbolically, $P=B G$. We do not propose to develop fully the relationships between the properties of $\phi, \psi$, 
and $\mathbf{X}$. Those relationships which will be developed in the next few lemmas are rather special ones which are essential for the proofs of our principal theorems.

LEMMA 3. Let $f(u)$ be a function continuous on the plane and let $\phi$ and $\psi$ be two set functions satisfying the hypotheses of Lemma 2. Then if $\mathrm{X}(A)$ is defined by (9), we have

$$
\iint_{B} f(u) d \mathrm{X}(u)=\iint_{E_{2}}\left(\iint_{(-v ; B)} f(u+v) d \phi(u)\right) d \psi(v)
$$

for all bounded sets $B$.

If in the plane we draw lines parallel to the coordinate axes at distance $1 / n$ apart, we induce a partition $\Pi_{n}$ of the set $B$ into a finite number of sets $B_{i}^{(n)}, i=1, \cdots, M_{n}$. By assigning boundaries properly we can take the sets $B_{i}^{(n)}$ to be pairwise disjoint. If we now fix $n$ for the moment and in each set $B_{i}^{(n)}$ select a point $u^{(i)}$, we get a simple function $F_{n}=\sum_{i=0}^{M_{n}} f\left(u^{(i)}\right) \theta_{i n}$, where $\theta_{i n}$ is the characteristic function of the set $B_{\imath}^{(n)}$. The sequence of simple functions so obtained clearly converges uniformly to $f(u)$ on the set $B$. Thus

$$
\begin{aligned}
& \iint_{B} f(u) d \mathrm{X}=\lim _{n \rightarrow \infty} \sum_{i=0}^{M_{n}} f\left(u^{(i)}\right) \mathrm{X}\left(B_{i}^{(n)}\right) \\
& =\lim _{n \rightarrow \infty} \sum_{i=0}^{M_{n}} f\left(u^{(i)}\right)\left\{\iint_{E_{2}} \phi\left(-v ; B_{i}^{(n)}\right) d \psi(v)\right\} \\
& =\lim _{n \rightarrow \infty} \iint_{E_{2}}\left\{\sum_{i=0}^{M_{n}} f\left(\bar{u}^{(i)}+v\right) \phi\left(-v ; B_{i}^{(n)}\right)\right\} d \psi(v),
\end{aligned}
$$

where $\bar{u}^{(i)}=u^{(i)}-v$. Recalling that $u^{(i)}$ is a point of $B_{i}^{(n)}$, we see that $\bar{u}^{(i)}$ is a point of $\left(-v ; B_{\imath}^{(n)}\right)$, and so for fixed $v$,

$$
\lim _{n \rightarrow \infty} \sum_{i=0}^{M_{n}} f\left(\bar{u}^{(i)}+v\right) \phi\left(-v ; B_{i}^{(n)}\right)=\iint_{(-v ; B)} f(u+v) d \phi(u) .
$$

Since $\bar{u}^{(i)}+v$ is always a point of the bounded set $B$ and since $f(u)$ is continuous, there is a constant $C$ such that $\left|f\left(\bar{u}^{(i)}+v\right)\right| \leqq C$ for all $\bar{u}^{(i)}+v$. Therefore, for fixed $n$ and $v$ the sum in (15) is majorized by

$$
C \sum_{i=0}^{M_{n}}\left|\phi\left(-v ; B_{i}^{(n)}\right)\right| \leqq C W(\phi ;(-v ; B)) \leqq C K_{B}
$$

where $K_{B}$ is as in Lemma 2. Hence, in view of the hypothesis that $\iint_{E_{2}}|d \psi|$ $<\infty$ we conclude that we may interchange the process of integration and passage to the limit in (14) and then from (15) obtain that

$$
\iint_{B} f(u) d \mathrm{X}(u)=\iint_{E_{2}}\left(\iint_{(-v ; B)} f(u+v) d \phi(u)\right) d \psi(v) .
$$


Lemma 4. Suppose $I_{1}=\int_{-\infty}^{+\infty} \int_{-\infty}^{+\infty} e^{i x u} d \Phi(u)$ and $I_{2}=\int_{-\infty}^{+\infty} \int_{-\infty}^{+\infty} e^{i x u} \gamma(u) d u$ where $\gamma(u)$ is bounded on $E_{2}$ and $\iint_{E_{2}}|d \Phi|<\infty$. Then the product $I_{1} I_{2}$ exists, equals $I_{2} I_{1}$, and the function $\mathrm{X}(A)$ in this case is absolutely continuous. Furthermore, if $\gamma(u)$ is continuous, then $\mathrm{X}(A)$ has a continuous derivative at every point of $E_{2}$.

We set $\Gamma(A)=\iint_{A} \gamma(s) d s$ and observe that

$$
\Gamma(-u ; A)=\iint_{(-u ; A)} \gamma(s) d s=\iint_{A} \gamma(-u+s) d s .
$$

Consequently $|\Gamma(-u ; A)| \leqq C|A|$, where $|A|$ denotes the Lebesgue measure of $A$ and $C$ is the bound of $\gamma$. Identifying $\Gamma$ with $\phi$ of Lemma 2 and $\Phi$ with $\psi$ of Lemma 2, we get that the product of $I_{1}$ and $I_{2}$ exists and that

$$
\mathrm{X}(A)=\iint_{E_{2}}\left(\iint_{(-u ; A)} \gamma(s) d s\right) d \Phi(u)=\iint_{E_{2}} \Phi(-u ; A) \gamma(u) d u .
$$

Rewriting the first integral and then applying Fubini's theorem gives

$$
\begin{aligned}
\mathrm{X}(A) & =\iint_{E_{2}}\left\{\iint_{A} \gamma(-u+s) d s\right\} d \Phi(u) \\
& =\iint_{A} d s \iint_{E_{2}} \gamma(-u+s) d \Phi(u) .
\end{aligned}
$$

From (16) it is clear that $\mathrm{X}(A)$ is absolutely continuous and has a derivative $D \mathbf{X}(x)$ almost everywhere, with

$$
D \mathrm{X}(x)=\iint_{E_{2}} \gamma(-u+x) d \Phi(u) .
$$

Whenever $\gamma(u)$ is continuous, the function $\iint_{E_{2}} \gamma(-u+x) d \Phi(u)$ will be a continuous function of $x$, as can readily be verified. Hence, in that case $D \mathbf{X}(x)$ will exist everywhere and will be continuous since $\mathbf{X}(A)$ is the indefinite integral of a continuous function.

2. Circular convergence. In this section we study trigonometric integrals (6) considered in the sense of circular convergence; i.e. $\lim _{R \rightarrow \infty} \iint_{C_{R}} e^{i x u} d \phi(u)$. A Riemann formula from which localization theorems follow will be developed, but even more important is the use of this formula to get equiconvergence of double integrals with double series. The proof of the Riemann formula is accomplished by means of formal products in a manner analogous to that used in the study of series.

\subsection{Multiplication theorems.}

TheOREM 1. Let $B$ and $G$ be two double trigonometric integrals as in (7). Suppose that $\phi$ satisfies the condition 


$$
\iint_{C_{R+1}} \Theta_{C_{R}}|d \phi|=o(1)
$$

and that

$$
\iint_{E_{2}}|u||d \psi|<\infty, \quad \lambda(x)=\iint_{E_{2}} e^{i x u} d \psi(u) .
$$

Then the formal product of $B$ and $G$ is defined,

and the difference

$$
P=\int_{-\infty}^{+\infty} \int_{-\infty}^{+\infty} e^{i x u} d \mathrm{X}(u)
$$

$$
\Delta_{R}(x)=\iint_{C_{R}} e^{i x u} d \mathrm{X}(u)-\lambda(x) \iint_{C_{R}} e^{i x u} d \phi(u)
$$

tends to zero as $R$ becomes infinite, uniformly in $x$.

To prove the existence of the product, we first note that for any bounded set $A$ there exists a positive number $a$, depending only on $A$, such that for any $u$ the set $(-u ; A)$ is contained in $C_{r+a} \ominus C_{r}$, where $r$ is positive and depends on $u$. Therefore in view of (18),

$$
|\phi(-u ; A)| \leqq \iint_{C_{r+a} \ominus_{C_{r}}}|d \phi(u)|=o(1) a \leqq K_{A} .
$$

Since $\iint_{E_{2}}|d \psi|<\infty$, the conditions of Lemma 2 are satisfied and hence $P$ is defined.

In proving that $\Delta_{R}(x) \rightarrow 0$ we first suppose that $x=0$. Then we may write

$$
\begin{aligned}
\Delta_{R} & =\iint_{C_{R}} d \mathrm{X}(u)-\lambda(0) \iint_{C_{R}} d \phi(u)=\mathrm{X}\left(C_{R}\right)-\lambda(0) \phi\left(C_{R}\right) \\
& =\iint_{E_{2}}\left\{\phi\left(-u ; C_{R}\right)-\phi\left(C_{R}\right)\right\} d \psi(u),
\end{aligned}
$$

the last equality following from (9) and (19). From (19) it also follows that we shall have proved the theorem for $x=0$ if we can show that given an $\epsilon>0$,

$$
\left|\phi\left(-u ; C_{R}\right)-\phi\left(C_{R}\right)\right| \leqq \epsilon C(|u|+1),
$$

where $C$ is a constant independent of $u$ and $R$ is sufficiently large.

If $|u| \geqq 2 R$, we have

$$
\begin{aligned}
\left|\phi\left(-u ; C_{R}\right)-\phi\left(C_{R}\right)\right| & \leqq\left|\phi\left(-u ; C_{R}\right)\right|+\left|\phi\left(C_{R}\right)\right| \\
& \leqq W\left(\phi ;\left(-u ; C_{R}\right)\right)+W\left(\phi ; C_{R}\right) \\
& \leqq W\left(\phi ; C_{|u|+R} \theta C_{|u|-R}\right)+W\left(\phi ; C_{R}\right) .
\end{aligned}
$$


Using (18) we see that the last sum is $o(1)\{(R+|u|)-(|u|-R)\}+o(1) R$ $=o(R)$, and since $|u| \geqq 2 R$, we see that for such $u$, (21) holds for $R$ sufficiently large. When $R<|u|<2 R$, we have

$$
\begin{aligned}
\left|\phi\left(-u ; C_{R}\right)-\phi\left(C_{R}\right)\right| & \leqq W\left(\phi ; C_{R+|u|} \ominus C_{R}\right)+W\left(\phi ; C_{R}\right) \\
& =o(R+|u|-R)+o(R)=o(|u|) .
\end{aligned}
$$

The first equality follows from (18) and the second from the fact that $R<|u|<2 R$. For $1 \leqq|u| \leqq R$, we have

$$
\begin{aligned}
\left|\phi\left(-u ; C_{R}\right)-\phi\left(C_{R}\right)\right| & \leqq W\left(\phi ; C_{R+|u|} \ominus C_{R}\right)+W\left(\phi ; C_{R} \ominus C_{R-|u|}\right) \\
& =o(R+|u|-R)+o(R-(R-|u|))=o(|u|) .
\end{aligned}
$$

Again the first equality follows from (18). Finally, it is clear that by the same methods we get the desired result if $0 \leqq|u| \leqq 1$.

We now pass to the proof for general $x$. By Lemma 3 we may write

$$
\iint_{C_{R}} e^{i x u} d \mathrm{X}(u)=\iint_{E_{2}}\left(\iint_{\left(-v ; C_{R}\right)} e^{i x u} d \phi(u)\right) e^{i x v} d \psi(v) .
$$

Defining

$$
\phi_{x}(E)=\iint_{E} e^{i x u} d \phi(u)
$$

we can rewrite the right-hand side of $(20)$ as

$$
\begin{aligned}
\iint_{E_{2}} e^{i x v} \phi_{x}\left(-v ; C_{R}\right) d \psi(v)-\lambda(x) \phi_{x}\left(C_{R}\right) & \\
& =\iint_{E_{2}} e^{i x v}\left\{\phi_{x}\left(-v ; C_{R}\right)-\phi_{x}\left(C_{R}\right)\right\} d \psi(v) .
\end{aligned}
$$

As before it is clear that to prove the theorem it suffices to show that given an $\epsilon>0$ for $R$ sufficiently large,

$$
\left|\phi_{x}\left(-u ; C_{R}\right)-\phi_{x}\left(C_{R}\right)\right| \leqq \epsilon C(|u|+1),
$$

where $C$ is a constant independent of $u, x$, and $R$. If we can establish that

$$
\iint_{C_{R+1} \Theta_{C R}}\left|d \phi_{x}\right|=o(1) \quad \text { as } R \rightarrow \infty,
$$

uniformly in $x$, then an analysis similar to that used to prove (21) will also yield (23). But to validate (24) is easy, for if $A$ is any finite union of disjoint sets $A_{j}$ contained in $C_{R+1} \ominus C_{R}$, we have

$$
\sum_{j=1}^{n}\left|\phi_{x}\left(A_{j}\right)\right|=\sum_{j=1}^{n}\left|\iint_{A_{j}} e^{i x u} d \phi(u)\right| \leqq \sum_{j=1}^{n} W\left(\phi ; A_{j}\right) \leqq W\left(\phi ; C_{R+1} \ominus C_{R}\right),
$$


and this implies that (24) holds uniformly in $x$.

Given a trigonometric integral $T=\int_{-\infty}^{+\infty} \int_{-\infty}^{+\infty} e^{i x u} d \phi(u)$, we define formal partial derivative operators in the obvious manner; namely

$$
\frac{\partial^{j+k}}{\partial x_{1}^{j} \partial x_{2}^{k}} T=\int_{-\infty}^{+\infty} \int_{-\infty}^{+\infty}(i)^{j+k}\left(u_{1}\right)^{j}\left(u_{2}\right)^{k} e^{i x u} d \phi(u), \quad j, k=0,1,2, \cdots .
$$

Another formal operation which we shall use is that of taking formal Laplacians. We define

$$
\nabla^{2} T=\frac{\partial^{2} T}{\partial x_{1}^{2}}+\frac{\partial^{2} T}{\partial x_{2}^{2}}=-\int_{-\infty}^{+\infty} \int_{-\infty}^{+\infty}|u|^{2} e^{i x u} d \phi(u) .
$$

It is not too difficult to show that whenever all integrals involved exist, these operators applied to products behave as they should. We prove this only for the simple cases which concern us.

LEMмA 5. If $B$ and $G$ are two trigonometric integrals as in (7) and

$$
\iint_{E_{2}}|u|^{2}|d \psi(u)|<\infty, \quad \iint_{C_{R+1}} \Theta_{C_{R}}|d \phi|=o\left(R^{-2}\right),
$$

then the formal product $P=B G$ exists and

$$
\nabla^{2} P=\left(\nabla^{2} B\right) G+2\left(\frac{\partial B}{\partial x_{1}} \frac{\partial G}{\partial x_{1}}+\frac{\partial B}{\partial x_{2}} \frac{\partial G}{\partial x_{2}}\right)+B \nabla^{2} G
$$

where the products on the right are formal.

Since the integral is an additive operator and formal multiplication is distributive with respect to addition, it is enough to show that

$$
\frac{\partial^{2} P}{\partial x_{i}^{2}}=\left(\frac{\partial^{2} B}{\partial x_{i}^{2}}\right) G+2\left(\frac{\partial B}{\partial x_{i}}\right)\left(\frac{\partial G}{\partial x_{i}}\right)+B\left(\frac{\partial^{2} G}{\partial x_{i}^{2}}\right) ; \quad i=1,2 .
$$

For the sake of definiteness we take $i=1$. Put

$$
\phi_{k}(A)=\iint_{A} u_{1}^{k} d \phi(u), \quad \psi_{k}(A)=\iint_{A} u_{1}^{k} d \psi(u), \quad k=0,1,2 .
$$

The functions of set $\phi_{k}$ and $\psi_{k}$ so defined obviously satisfy conditions 1 (a) and 1 (b) and furthermore,

$$
\iint_{C_{R_{1}}} \Theta_{C_{R}}\left|d \phi_{k}\right|=o(1), \quad \iint_{E_{2}}\left|d \psi_{k}\right|<\infty .
$$

The last two statements are proved as follows. First note that $\iint_{E_{2}}\left|d \psi_{k}\right|$ $\leqq \iint_{E_{2}}|u|^{2}|d \psi|<\infty$. Secondly, if $A$ is the finite union of disjoint sets $A_{i}$ contained in $C_{R+1} \ominus C_{R}$, then 


$$
\sum_{i=1}^{n}\left|\phi_{k}\left(A_{i}\right)\right| \leqq \iint_{C_{R+1} \Theta_{C R}}(R+1)^{2}|d \phi(u)|=(R+1)^{2} o\left(R^{-2}\right)=o(1)
$$

and so $W\left(\phi_{k} ; C_{R+1} \ominus C_{R}\right)=o(1)$. Thus each term on the right-hand side of (25) makes sense since it is the product of integrals of the type $\int_{-\infty}^{+\infty} \int_{-\infty}^{+\infty} e^{i x u} d \phi_{k}(u)$ by $\int_{-\infty}^{+\infty} \int_{-\infty}^{+\infty} e^{i x u} d \psi_{2-k}, k=0,1,2$. We may write the righthand side of $(25)$ as $\int_{-\infty}^{+\infty} \int_{-\infty}^{+\infty} e^{i x u} d \mathrm{X}_{*}(u)$, where

$$
\begin{aligned}
\mathrm{X}_{*}(A) & =(i)^{2} \iint_{E_{2}}\left\{\phi_{2}(-u ; A)+2 u_{1} \phi_{1}(-u ; A)+u_{1}^{2} \phi(-u ; A)\right\} d \psi(u) \\
& =(i)^{2} \iint_{E_{2}}\left\{\iint_{(-u ; A)}\left(v_{1}^{2}+2 u_{1} v_{1}+u_{1}^{2}\right) d \phi(v)\right\} d \psi(u) \\
& =(i)^{2} \iint_{E_{2}}\left\{\iint_{(-u ; A)}\left(v_{1}+u_{1}\right)^{2} d \phi(v)\right\} d \psi(u) .
\end{aligned}
$$

On the left-hand side of (25) we have $\int_{-\infty}^{+\infty} \int_{-\infty}^{+\infty} e^{i x u} d \mathrm{X}^{\prime}(u)$, where $\mathrm{X}^{\prime}(A)$ $=(i)^{2} \iint_{A} v_{1}^{2} d \mathrm{X}(v)$. Applying Lemma 3 to this integral shows that

$$
\mathrm{X}^{\prime}(A)=(i)^{2} \iint_{E_{2}}\left(\iint_{(-u ; A)}\left(v_{1}+u_{1}\right)^{2} d \phi(v)\right) d \psi(u) .
$$

Comparing this with the last integral in (26), we get that $\mathrm{X}^{\prime}(A)=\mathrm{X}_{*}(A)$, and so (25) is established.

2.2. Localization and equiconvergence theorems. Before developing the theorems of this section we discuss several notions which are indispensable for our purposes.

If $F(s)$ is integrable over the plane, then it has a Fourier transform

$$
\gamma(u)=\frac{1}{4 \pi^{2}} \iint_{E_{2}} F(s) e^{-i s u} d s .
$$

We shall consider the circular partial Fourier integrals

$$
S_{R}(x)=\iint_{C_{R}} \gamma(u) e^{i x u} d u
$$

Defining

$$
D_{R}(x)=\iint_{C_{R}} e^{i x u} d u=D_{R}(-x)
$$

we may write

$$
S_{R}(x)=\frac{1}{4 \pi^{2}} \iint_{E_{2}} F(s) D_{R}(x-s) d s
$$


Finally, we have

$$
\frac{\partial^{j+k}}{\partial x_{1}^{j} \partial x_{2}^{k}} S_{R}(x)=\frac{1}{4 \pi^{2}} \iint_{E_{2}} F(s) \frac{\partial^{j+k}}{\partial x_{1}^{j} \partial x_{2}^{k}} D_{R}(x-s) d s .
$$

In particular,

$$
\nabla^{2} S_{R}(x)=\frac{1}{4 \pi^{2}} \iint_{E_{2}} F(s) \nabla^{2} D_{R}(x-s) d s
$$

where in the evaluation of $\nabla^{2} D_{R}(x-s)$ we may differentiate either with respect to $\left(x_{1}, x_{2}\right)$ or with respect to $\left(s_{1}, s_{2}\right)$.

A notion of which we shall make much use is that of a localizing function. By a domain we shall mean an open connected set, and by a closed domain we shall mean a domain plus its boundary. If $D_{1}$ is a closed bounded domain and $D$ is a closed bounded domain contained in $D_{1}^{\circ}$, the interior of $D_{1}$, then we may construct a function which is equal to one on $D$, zero outside of $D_{1}$, and has as many derivatives as we please. A construction for such a function is given in [1].

We now define an anti-Laplacian operator, which we denote by $\nabla^{-2}$, to act on trigonometric integrals as follows. Given a trigonometric integral $B$ as in (7), we define

$$
\begin{aligned}
\nabla^{-2} B= & \frac{\left(x_{1}+x_{2}\right)^{2} \phi(0)}{2}+\iint_{C_{1}-(0)} L(x, u) d \phi(u) \\
& -\iint_{|u|>C_{1}} \frac{e^{i x u}}{|u|^{2}} d \phi(u),
\end{aligned}
$$

where (0) denotes the set consisting of the origin and

$$
L(x, u)=-\frac{e^{i x u}-1-i x u}{(i|u|)^{2}}=\sum_{k=2}^{\infty} \frac{(i)^{k}\left(x_{1} u_{1}+x_{2} u_{2}\right)^{k}}{k !\left(u_{1}^{2}+u_{2}^{2}\right)}, \quad u \neq 0 .
$$

It is easy to see that subject to the restriction $u \neq 0$ the series on the right converges uniformly for $x$ and $u$ each lying in bounded sets. Moreover, $L(x, u)$ is regular in $x_{1}$ and $x_{2}$, and for $u$ and $x$ each in bounded sets, the derivatives of $L(x, u)$ with respect to $x_{1}$ and $x_{2}$ are bounded. Returning to formula (27) we see that the function $\phi(0)\left(x_{1}+x_{2}\right)^{2} / 2$ plus the first integral on the right represent a function $F_{1}(x)$ which is of class $C^{(\infty)}$. If the function $\phi$ satisfies the condition $W\left(\phi ; C_{R+1} \ominus C_{R}\right)=O(1)$, the second integral in (27) converges uniformly in $x$ and hence represents a continuous function $F_{2}(x)$. The function $F(x)=F_{1}(x)+F_{2}(x)$ is called the Riemann function for the integral $B$.

We can now deduce the Riemann formula, which contains the localization principle for trigonometric integrals.

THEOREM 2. Let $B=\int_{-\infty}^{\infty} \int_{-\infty}^{\infty} e^{i x u} d \phi(u)$ where $W\left(\phi ;\left(-u ; C_{1 / 2}\right)\right)=o\left(|u|^{-1}\right)$. 
Let $\lambda(x)$ be a localizing function which is of class $C^{(10)}$ and is associated with the closed bounded domains $D$ and $D_{1}, D \subset D_{1}^{\circ}$. Then if $F(x)$ is the Riemann function for $B$, the difference

$$
\Delta_{R}(x)=\iint_{C_{R}} e^{i x u} d \phi(u)-\frac{1}{4 \pi^{2}} \iint_{E_{2}} F(u) \lambda(u) \nabla^{2} D_{R}(x-u) d u
$$

tends to zero as $R \rightarrow \infty$, uniformly for $x$ in $D$.

Before giving the proof of the theorem we observe that the condition $W\left(\phi ;\left(-u ; C_{1 / 2}\right)\right)=o\left(|u|^{-1}\right)$ implies $W\left(\phi ; C_{R+1} \ominus C_{R}\right)=o(1)$. The theorem remains true for this weaker condition, for as will be seen from the proof, the fact that $W\left(\phi ; C_{R+1} \ominus C_{R}\right)=o(1)$ is, except for one point, the condition which is actually used. Modification of the proof to accommodate the case $W\left(\phi ; C_{R+1} \ominus C_{R}\right)=o(1)$ will be indicated at the proper place.

Our proof will first be carried out under the assumption that $W\left(\phi ; C_{1}\right)=0$. The Riemann function is therefore given by

$$
F(x)=-\iint_{E_{2}} \frac{e^{i x u}}{|u|^{2}} d \phi(u) .
$$

If we set

$$
\Phi(A)=\iint_{A} \frac{d \phi(u)}{|u|^{2}}
$$

we may designate the integral defining $F(x)$ as

$$
\Im=-\iint_{E_{2}} e^{i x u} d \Phi(u)=F(x) .
$$

Since the function $\lambda(x)$ is integrable on the plane, it has a Fourier transform

$$
\gamma(u)=\frac{1}{4 \pi^{2}} \iint_{E_{2}} e^{-i x u} \lambda(x) d x,
$$

and since $\lambda$ is of class $C^{(10)}$, integration by parts shows that $\gamma(u)$ $=o\left(1 /\left(\left|u_{1}\right|+1\right)^{5}\right) o\left(1 /\left(\left|u_{2}\right|+1\right)^{5}\right)=o\left(1 /|u|^{5}\right)$. Thus the function $\gamma(u)$ is integrable over the plane. This fact plus the fact that $\lambda(x)$ is continuous enable us to apply standard inversion theorems and get that

$$
\lambda(x)=\iint_{E_{2}} e^{i x u} \gamma(u) d u .
$$

The integral on the right is the Fourier integral of $\lambda$ and shall be designated by $\mathfrak{F}[\lambda]$. Also, in view of the fact that $\lambda(x)$ is of class $C^{(10)}$, we have 


$$
\frac{\partial^{i+j}}{\partial x_{1}^{i} \partial x_{2}^{j}} \mathfrak{F}[\lambda]=\mathfrak{F}\left[\frac{\partial^{i+j}}{\partial x_{1}^{i} \partial x_{2}^{j}} \lambda(x)\right],
$$

$i, j=0,1,2 ; i+j \leqq 2$. The integral on the right actually converges absolutely and uniformly in $x$ to $\partial^{i+i} \lambda(x) / \partial x_{1}^{i} \partial x_{2}^{j}$.

If we set

$$
\Gamma(A)=\iint_{A} \gamma(u) d u
$$

then we may write

$$
\lambda(x)=\mathfrak{F}[\lambda]=\iint_{E_{2}} e^{i x u} d \Gamma(u) .
$$

From the fact that $\gamma(u)=o\left(|u|^{-5}\right)$ it is readily seen that $\iint_{E_{2}}|u|^{2}|d \Gamma(u)|<\infty$. Also, if $W\left(\phi ; C_{R+1} \ominus C_{R}\right)=o(1)$, it is clear that $W\left(\Phi ; C_{R+1} \ominus C_{R}\right)=o\left(R^{-2}\right)$. Hence, by Lemma $5, \mathfrak{B}=\mathfrak{\Im} \mathfrak{F}[\lambda]$ exists and

$$
\nabla^{2} \mathfrak{B}=\left(\nabla^{2} \Im\right) \mathfrak{F}[\lambda]+2\left(\frac{\partial \mathfrak{Y}}{\partial x_{1}} \frac{\partial \mathfrak{F}}{\partial x_{1}}+\frac{\partial \mathfrak{Y}}{\partial x_{2}} \frac{\partial \mathfrak{F}}{\partial x_{2}}\right)+\Im\left(\nabla^{2} \mathfrak{F}\right) .
$$

By (30) each of the integrals $\partial \mathfrak{F} / \partial x_{1}, \partial \mathfrak{F} / \partial x_{2}$, and $\nabla_{2} \varsubsetneqq$ converges absolutely and is also uniformly convergent to zero for $x$ in $D$. Each of the integrals $\partial \Im / \partial x_{1}, \partial \Im / \partial x_{2}$, and $\Im$ satisfies the condition that the total variation of the function with respect to which the integral is taken is $o(1)$ with $R$ on $C_{R+1}$ $\ominus C_{R}$. Hence, adopting the notation that $T_{R}$ shall designate the trigonometric integral $T$ taken over $C_{R}$, we get from Theorem 1 that

$$
\left\{\nabla^{2} \mathfrak{B}-\left(\nabla^{2} \Im\right) \mathfrak{F}[\lambda]\right\}_{R} \rightarrow 0
$$

as $R \rightarrow \infty$, uniformly for $x$ in $D$. But $\nabla^{2} \Im=B$, so another application of Theorem 1 and the fact that $\lambda(x)=1$ on $D$ yields

$$
\left(\nabla^{2} \mathfrak{B}\right)_{R}-B_{R} \rightarrow 0
$$

uniformly for $x$ in $D$.

To complete the proof for the case $W\left(\phi ; C_{1}\right)=0$ we need only show that $\left(\nabla^{2} \mathfrak{B}\right)_{R}$ is equal to the second integral on the right in (29). If we set

$$
M(A)=\iint_{E_{2}} \Phi(-u ; A) d \Gamma(u)=\iint_{E_{2}} \Gamma(-u ; A) d \Phi(u),
$$

we may write

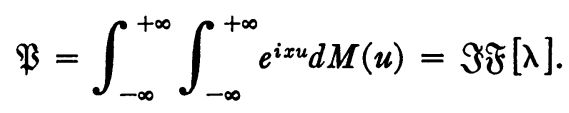

From Theorem 1 and from the known facts about $\mathfrak{F}$ and $\mathfrak{F}$ we obtain that 
$\mathfrak{P}$ converges to $F(x) \lambda(x)$ uniformly in $x$. If it can be shown that $M(A)$ is absolutely continuous with a continuous derivative $D M(x)=\mu(x)$ which is also integrable over the plane, then

$$
F(x) \lambda(x)=\iint_{E_{2}}^{i x u} \mu(u) d u .
$$

Since $F \lambda$ is integrable, this would give

$$
\mu(u)=\frac{1}{4 \pi^{2}} \iint_{E_{2}} F(x) \lambda(x) e^{-i x u} d x .
$$

In other words $\mu(u)$ would be the Fourier transform of $F \lambda$ and $\mathfrak{B}$ would be the Fourier integral of $F \lambda$. Hence

$$
\left(\nabla^{2} \mathfrak{B}\right)_{R}=\frac{1}{4 \pi^{2}} \iint_{E_{2}} F(u) \lambda(u) \nabla^{2} D_{R}(x-u) d u=\iint_{D_{1}},
$$

and substituting this in (31) would yield the desired result. Thus to prove the theorem we need only show that $\mu(u)$ exists everywhere, is continuous, and is integrable. This we do next.

Since $\gamma(u)$ is bounded and continuous and $\iint_{E_{2}}|d \Phi|<\infty$, Lemma 4 immediately yields the fact that $D M(x)=\mu(x)$ exists everywhere and is continuous. We now propose to show that

$$
\mu(x)=o\left(|x|^{-3}\right)
$$

and hence is integrable. Equation (17) tells us that $\mu(x)=\iint_{E_{2}} \gamma(-u+x) d \Phi(u)$ where now $W\left(\Phi ; C_{R+1} \ominus C_{R}\right)$ is $o\left(R^{-2}\right), \gamma(u)=o\left(|u|^{-5}\right)$. We next break up this integral as follows:

$$
\iint_{|u| \leqq|x| / 2}+\iint_{|x| / 2<|u|<3|x| / 2}+\iint_{|u| \geqq 3|x| / 2}=J+K+L .
$$

Now

$$
\begin{aligned}
|J| & \leqq\left\{\max _{|u| \leqq|x| / 2} \gamma(-u+x)\right\} \iint_{|u| \leqq|x| / 2}|d \Phi(u)| \\
& \leqq o\left(|x|^{-5}\right) \iint_{E_{2}}|d \Phi(u)|=o\left(|x|^{-5}\right)
\end{aligned}
$$

and

$$
|L| \leqq\left\{\max _{|u|>3|x| / 2} \gamma(-u+x)\right\} \iint_{E_{2}}|d \Phi(u)|=o\left(|x|^{-5}\right) .
$$

For the integral $K$ we have 


$$
|K| \leqq \sum_{n=[|x| / 2]}^{[3|x| / 2]+1} \iint_{A_{n}}|\gamma(-u+x)||d \Phi(u)|+\iint_{A_{x}}=K_{1}+K_{2}
$$

where by $[a]$ we mean the integral part of $a, A_{n}=C_{n+1} \ominus C_{n}, A_{x}=C_{[|x|]+2}$ $\ominus C_{[|x|]-2}$, and the prime indicates that the annulus $A_{x}$ is to be omitted from the summation. Let us now evaluate a typical integral

$$
I_{p}=\iint_{A_{p}}|\gamma(-u+x)||d \Phi(u)|, \quad \quad p \neq 0 .
$$

We begin by noting that while the vector $u$ runs through the annulus $A_{p}$, the vector $-u+x$ runs through an annulus $B_{p}$ with center at $x$ and radii $p, p+1$. The annulus $A_{p}$ can be broken up into $m(p)$ disjoint sets $\sigma_{i}, i=1,2, \cdots$, $m(p)$, each of diameter less than one-half, where $m(p)$ is an integer depending on $p$ and satisfying the inequality $m(p) \leqq p C, C$ a constant. Thus

$$
\begin{aligned}
I_{p} & \leqq \sum_{i=1}^{m(p)} \iint_{\sigma_{i}}|\gamma(-u+x)||d \Phi(u)| \\
& \leqq \sum_{i=1}^{m(p)} \max _{u \in \sigma_{i}}\{|\gamma(-u+x)|\} \iint_{\sigma_{i}}|d \Phi(u)| \\
& \leqq o\left(|p|^{-3}\right) \sum_{i=1}^{m(p)} \max _{u \in \sigma_{i}}\{|\gamma(-u+x)|\} \\
& \leqq o\left(|p|^{-3}\right) \sum_{j=[|x|-p]}^{\lfloor|x|+p]+1} 2 \pi j\left[\max \gamma(v) ; v \in A_{i}\right] \\
& =o\left(|p|^{-3}\right) o\left(\frac{1}{|| x|-p|^{3}}\right) .
\end{aligned}
$$

In a similar fashion we get that $K_{2}=o\left(|x|^{-3}\right) O(1)$. Using this and the estimate obtained for $I_{p}$, we get from (35) that $K=o\left(|x|^{-3}\right)$. Thus $\mu(x)=o\left(|x|^{-3}\right)$ and the theorem is proved when $W\left(\phi ; C_{1}\right)=0$.

Had we been given the condition $W\left(\phi ; C_{R+1} \ominus C_{R}\right)=o(1)$, we could not conclude that $\mu(x)=o\left(|x|^{-3}\right)$. However, by methods similar to those used here we could show that $W\left(M ; C_{R+1} \ominus C_{R}\right)=o\left(R^{-2}\right)$, and this would show that $\mu(u)$ is integrable over the plane. Although this last fact is all that is required for the proof of the present theorem, we insisted on the estimate $\mu(x)=o\left(|x|^{-3}\right)$ because we shall need it to prove Theorem 3 below.

Suppose now that $\phi(E)=0$ for any set $E$ exterior to the unit circle. The Riemann function corresponding to $B$ will now be the regular function

$$
F(x)=\iint_{C_{1}-(0)} L(x, u) d \phi(u)+\frac{\left(x_{1}+x_{2}\right)^{2}}{2} \phi(0),
$$

where $L(x, u)$ is defined in (28). Our task now is to prove that the difference 


$$
\Delta_{R}(x)=\iint_{C_{1}} e^{i x u} d \phi(u)-\frac{1}{4 \pi^{2}} \int_{-\infty}^{+\infty} \int_{-\infty}^{+\infty} F(u) \lambda(u) \nabla^{2} D_{R}(x-u) d u
$$

tends to zero as $R$ tends to infinity, uniformly for $x$ in $D$. Recalling that $\lambda(u)$ vanishes outside of $D_{1}$, we see that the second integral in (37) is actually extended only over $D_{1}$. Thus, we can apply Fubini's theorem and then integrate by parts to transform this integral into

$$
\int_{-\infty}^{+\infty} \int_{-\infty}^{+\infty} \nabla^{2}\{F(u) \lambda(u)\} D_{R}(x-u) d u .
$$

The function $F \lambda$ being of class $C^{(10)}$, the Fourier integral of $\nabla^{2} F \lambda$ will be absolutely and uniformly and hence circularly and uniformly convergent to $\nabla^{2} F \lambda$. For $x$ in $D, \nabla^{2} F \lambda=\nabla^{2} F$, and so

$$
\nabla^{2} F(x)=\iint_{C_{1}} e^{i x u} d \phi(u),
$$

Hence the difference in (37) tends to zero as $R$ becomes infinite, uniformly for $x$ in $D$.

Since any set function $\phi(E)$ is the sum of two functions, one with $\phi(E)=0$ for $E \subset C_{1}$ and the other satisfying $\phi(E)=0$ for $E$ exterior to $C_{1}$, the theorem is proved.

In the next theorem we shall designate by $\Omega$ the fundamental square bounded by the lines $x_{1}=0, x_{1}=2 \pi, x_{2}=0, x_{2}=2 \pi$. We shall also have occasion to deal with double trigonometric series and shall use the single index notation for them in accordance with the rules laid down in the introduction.

THEOREM 3. Let $B=\int_{-\infty}^{+\infty} \int_{-\infty}^{+\infty} e^{i x u} d \phi(u)$ be a double trigonometric integral such that $W\left(\phi ;\left(-u ; C_{1 / 2}\right)\right)=o\left(|u|^{-1}\right)$. If $D^{*}$ is a closed bounded domain contained in the interior of $\Omega$, then there is a double trigonometric series $\sum a_{m} e^{i m x}$ with coefficients $a_{m}=o\left(|m|^{-1}\right)$ such that

$$
\iint_{C R} e^{i x u} d \phi(u)-\sum_{|m| \leqq R} a_{m} e^{i m x}
$$

tends to zero as $R \rightarrow \infty$, uniformly for $x$ in $D^{*}$.

For integrals of the form

$$
g(x)=\iint_{C_{1}} e^{i x u} d \phi(u)
$$

the result is almost immediate. Let $D$ be a bounded closed domain interior to $\Omega$ and containing $D^{*}$ in its interior. Let $\lambda$ be a sufficiently good localizing function associated with the domains $D$ and $D^{*}$. Since $g(x)$ is regular, the function $h(x)$ which equals $g(x) \lambda(x)$ on $\Omega$ and is periodic can be expanded in 
a double Fourier series $\subseteq[h]$ which converges uniformly to $g(x)$ on $D^{*}$. Therefore, if we can prove the theorem for the case $W\left(\phi ; C_{1}\right)=0$, we are through.

We now assume $W\left(\phi ; C_{1}\right)=0$. Let $D$ and $D_{1}$ be two closed bounded domains such that $D^{*} \subset D^{\circ} \subset D \subset D_{1}^{\circ} \subset D_{1} \subset \Omega$. If $\lambda$ is the localizing function associated with the domains $D^{*}$ and $D$, then from Theorem 2 and its proof we get that

$$
\left\{\iint_{C_{R}} e^{i x u} d \phi(u)-\frac{1}{4 \pi^{2}} \iint_{D} F(u) \lambda(u) \nabla^{2} D_{R}(x-u) d u\right\} \rightarrow 0
$$

as $R$ becomes infinite, uniformly for $x$ in $D^{*}$. We also get

$$
\begin{aligned}
& \mu(u)=\frac{1}{4 \pi^{2}} \int_{0}^{2 \pi} \int_{0}^{2 \pi} F(x) \lambda(x) e^{-i x u} d x=\frac{1}{4 \pi^{2}} \iint_{E_{2}}, \\
& \mu(u)=o\left(|u|^{-3}\right) .
\end{aligned}
$$

Let us define a function $G(x)$ to be equal to $F(x) \lambda(x)$ for $x$ in $\Omega$ and to be periodic of period $2 \pi$ in each variable $x_{1}$ and $x_{2}$. Then we may develop $G(x)$ in its double Fourier series

$$
\Im[G]=a_{00}+\sum^{\prime} \frac{a_{m}}{|m|^{2}} e^{i m x}
$$

where the prime indicates that the term $a_{00}$ is omitted. Equations $\left(33^{\prime}\right)$ and $\left(34^{\prime}\right)$ of the present theorem tell us that the coefficients $a_{m}$ are $o\left(|m|^{-1}\right)$, and hence $\subseteq[G]$ converges uniformly to $G(x)$.

We now study the series

$$
\sum^{\prime} a_{m} e^{i m x}
$$

which is obtained from (39) by formally applying the Laplacian operator termwise. It can be written as a double trigonometric integral in the following fashion. Denote by $\Lambda$ the set of lattice points $m=\left(m_{1}, m_{2}\right)$ in the plane, with the point $(0,0)$ excluded from $\Lambda$. Define a set function $\theta$ by setting $\theta(m)$ $=a_{m}$ for $|m| \neq 0, \theta(\mathrm{U}(m))=\sum a_{m}$ for all finite collections of lattice points, and $\theta(E)=\theta(E \wedge \Lambda)$. Then clearly (40) can be written as the integral

$$
\int_{-\infty}^{+\infty} \int_{-\infty}^{+\infty} e^{i x u} d \theta(u)
$$

with $W\left(\theta ;\left(-u ; C_{1 / 2}\right)\right)=o\left(|u|^{-1}\right)$. Also, it is evident that the Riemann function for (41) is $G(x)-a_{00}$. Hence, if $\lambda_{1}$ is the localizing function associated with the domains $D$ and $D_{1}$, Theorem 2 tells us that

$$
\left\{\iint_{C_{R}} e^{i x u} d \theta(u)-\frac{1}{4 \pi^{2}} \iint_{E_{z}}\left[G(u)-a_{00}\right] \lambda_{1}(u) \nabla^{2} D_{R}(x-u) d u\right\} \rightarrow 0
$$


as $R \rightarrow \infty$, uniformly for $x$ in $D$. Now,

$$
\frac{1}{4 \pi^{2}} \iint_{E_{2}} \nabla^{2}\left\{a_{00} \lambda_{1}(u)\right\} D_{R}(x-u) \rightarrow 0
$$

as $R \rightarrow \infty$, uniformly for $x$ in $D$. Using this fact and the fact that $G(u)$ $=F(u) \lambda(u)$ on $\Omega$, we obtain from (42) that

$$
\left\{\iint_{C R} e^{i x u} d \theta(u)-\frac{1}{4 \pi^{2}} \iint_{D_{1}}[F(u) \lambda(u)] \lambda_{1}(u) \nabla^{2} D_{R}(x-u)\right\} \rightarrow 0
$$

as $R \rightarrow \infty$, uniformly for $x$ in $D$. But $\lambda(u)=0$ outside of $D$ and $\lambda_{1}(u)=1$ on $D$, so (43) can be written as

$$
\left\{\iint_{C_{R}} e^{i x u} d \theta(u)-\frac{1}{4 \pi^{2}} \iint_{D} F(u) \lambda(u) \nabla^{2} D_{R}(x-u)\right\} \rightarrow 0
$$

as $R \rightarrow \infty$, uniformly for $x$ in $D$. Recalling that the first integral in (44) is actually the series (40), we see that a comparison of (44) with (29) of the present theorem completes the proof.

THEOREM 4. Let $B=\int_{-\infty}^{+\infty} \int_{-\infty}^{+\infty} e^{i x u} d \phi(u)$ be a trigonometric integral such that $\phi$ satisfies the condition $W\left(\phi ; C_{R+1} \ominus C_{R}\right)=o(1)$, and let $D$ be a closed bounded domain. Then there is a function $\alpha(u)$ which is continuous and such that

$$
\iint_{C_{R}} e^{i x u} d \phi(u)-\iint_{C_{R}} e^{i x u} \alpha(u) d u
$$

tends to zero as $R \rightarrow \infty$, uniformly for $x$ in $D$.

First let us suppose that $W\left(\phi ; C_{1}\right)=0$. Let $D_{1}$ be a closed bounded domain such that $D \subset D_{1}^{\circ}$ and let $\lambda(u)$ be a localizing function of class $C^{(10)}$ associated with $D$ and $D_{1}$. The proof now proceeds like the proof of Theorem 2 for the case $W\left(\phi ; C_{1}\right)=0$. Using the notation of that theorem we have

$$
\left\{\nabla^{2} \mathfrak{B}-B\right\}_{R} \rightarrow 0
$$

as $R \rightarrow \infty$, uniformly for $x$ in $D$, where

$$
\mathfrak{B}=\int_{-\infty}^{+\infty} \int_{-\infty}^{+\infty} e^{i x u} d M(u)=\int_{-\infty}^{+\infty} \int_{-\infty}^{+\infty} e^{i x u} \mu(u) d u
$$

and $\mu(x)$ is continuous, is given by (33), and satisfies (34). Thus (31) may be written as

$$
\iint_{C_{R}} e^{i x u}(i|u|)^{2} \mu(u) d u-\iint_{C_{R}} e^{i x u} d \phi(u) \rightarrow 0
$$

as $R \rightarrow \infty$, uniformly for $x$ in $D$. Identifying $\left(i|u|^{2}\right) \mu(u)$ with $\alpha(u)$ gives the theorem for the case in which $W\left(\phi ; C_{1}\right)=0$. 
If $\phi(E)=0$ for any $E$ outside of $C_{1}$,

$$
\int_{-\infty}^{+\infty} \int_{-\infty}^{+\infty} e^{i x u} d \phi(u)=\iint_{C_{1}} e^{i x u} d \phi(u)=F(x) .
$$

Clearly, $F(x)$ is regular. Let $\lambda(x)$ be a localizing function associated with the domains $D$ and $D_{1}$. The function $F \lambda$ will then be integrable over the plane and if $\lambda$ has enough derivatives the Fourier integral of $F, \int_{-\infty}^{+\infty} \int_{-\infty}^{+\infty} e^{i x u} \omega(u) d u$, where $\omega(u)$ is the continuous Fourier transform of $F \lambda$, will converge to $F \lambda$, uniformly for $x$ in $D$. But on $D, F(x) \lambda(x)=F(x)$ and this proves the theorem for functions $\phi$ such that $\phi(E)=0$ for $E$ outside of $C_{1}$. Combining the results for the two cases treated gives Theorem 4 .

2.3. Extensions to higher dimensions. If we denote the $k$-dimensional sphere of radius $R$ and center at the origin by $S_{R}^{(k)}$ and make the other obvious notational changes, then we may state the following extension of Theorem 3 .

THEOREM 5. Let $B=\int_{-\infty}^{+\infty} \cdots \int_{-\infty}^{+\infty} e^{i x u} d \phi(u)$ be a k-dimensional trigonometric integral, where $W\left(\phi ;\left(-u ; S_{1 / 2}^{(k)}\right)\right)$ is $o\left(|u|^{-k+1}\right)$ and $k \geqq 1$. Then given any closed domain $D$ contained in the interior of the k-dimensional fundamental cube $\Omega$, there is a trigonometric series $\sum a_{m} e^{i m x}$ such that $a_{m}=o\left(|m|^{-k+1}\right)$ and

$$
\int \cdots \int_{|u| \leqq R} e^{i x u} d \phi(u)-\sum_{|m| \leqq R} a_{m} e^{i m x}
$$

tends to zero as $R \rightarrow \infty$, uniformly for $x$ in $D$.

The proof of this theorem, except for the necessary modifications of the order conditions, is the same as that of Theorem 3. It is clear that we can also state $k$-dimensional theorems corresponding to Theorems 1,2 , and 4 . This we leave to the reader.

2.4. The conjugate integral. By the integral conjugate to a trigonometric integral $B$ we shall mean the integral

$$
\tilde{B}=\int_{-\infty}^{+\infty} \int_{-\infty}^{+\infty} \epsilon\left(u_{1}\right) \epsilon\left(u_{2}\right) e^{i x u} d \phi(u)
$$

where $\epsilon\left(u_{i}\right)=1$ if $u_{i}>0, \epsilon\left(u_{i}\right)=-1$ if $u_{i}<0$, and $\epsilon(0)=0, i=1,2$. In the onedimensional case results parallel to those obtained for a trigonometric integral are obtained for its conjugate. (See [5].) That it is not possible to deduce such results by our methods in the case of double integrals follows from known results on double trigonometric series. For the pertinent counterexample the reader is referred to [1].

3. Restricted convergence. We shall now study trigonometric integrals in the sense of restricted convergence and shall establish the analogues of Theorems 1-4 in this situation. In principle the proofs are similar to those employed in the circular case. Therefore, full details will be given only at 
points of difference and omissions will be made where the similarity with the circular case is strong.

The following special notations will be used frequently in this part of the paper. By $\left[b_{1}, b_{2}\right]$ or $[b]$ we shall mean the closed rectangle with center at the origin and bounded by the lines $x_{1}= \pm b_{1}, x_{2}= \pm b_{2} ; b_{1}, b_{2},>0$. If $A_{1}$ and $A_{2}$ are two fixed positive real numbers, then $A(b)=A\left(b_{1}, b_{2}\right)$ shall be used to denote the set $\left[b_{1}+1, b_{2}+1\right] \ominus\left[b_{1}, b_{2}\right]$, where $b_{1}$ and $b_{2}$ are subject to the restriction that $A_{1} \leqq b_{1} / b_{2} \leqq A_{2}$. Given a point $u \equiv\left(u_{1}, u_{2}\right)$, by $\|u\|$ we shall mean the maximum of $\left|u_{1}\right|$ and $\left|u_{2}\right|$. A function $f\left(u_{1}, u_{2}\right)$ is said to converge rapidly to a limit $l$ as $\left|u_{1}\right|$ and $\left|u_{2}\right|$ become infinite if given any $\epsilon>0$ a positive real number $R$ can be found with the property that $|f(u)-l|<\epsilon$ for all $u$ exterior to $[R, R]$.

3.1. Multiplication theorems. A set function $\phi$ will be said to satisfy condition $\mathrm{W}_{0}$ in case

$\mathrm{W}_{0}$ :

$$
W(\phi ;(u ;[1 / 2,1 / 2]))=o\left(\left(\left|u_{1}\right|+1\right)^{-1 / 2}\left(\left|u_{2}\right|+1\right)^{-1 / 2}\right) \text { rapidly. }
$$

It will be said to satisfy condition $\mathrm{W}_{0}^{*}$ in case for any preassigned positive numbers $A_{1}$ and $A_{2}$

$\mathrm{W}_{0}^{*}$

$$
W\left(\phi ; A\left(b_{1}, b_{2}\right)\right)=o(1) \text { rapidly }
$$

as $\left(b_{1}, b_{2}\right) \rightarrow \infty$. That is, given any $\epsilon>0$ we can find a positive number $R$ such that $W\left(\phi ; A\left(b_{1}, b_{2}\right)\right)<\epsilon$ whenever $b_{1}$ or $b_{2}$ is greater than $R$, yet subject to the restriction $A_{1} \leqq b_{1} / b_{2} \leqq A_{2}$. One can readily verify that the condition $\mathrm{W}_{0}$ implies $\mathrm{W}_{0}^{*}$.

Theorem 6. Let $B$ and $G$ be two trigonometric integrals as in (7). Suppose that $\phi$ satisfies condition $\mathrm{W}_{0}^{*}$ and that

$$
\begin{gathered}
\iint_{E_{2}}\left(\left|u_{1}\right|+1\right)\left(\left|u_{2}\right|+1\right)|d \psi(u)|<\infty ; \\
\lambda(x)=\iint_{E_{2}} e^{i x u} d \phi(u) .
\end{gathered}
$$

Then $P$, the formal product of $B$ and $G$, is well defined and the difference

$$
\Delta_{S_{1}, S_{2}}(x)=\int_{-S_{2}}^{S_{2}} \int_{-S_{1}}^{S_{1}} e^{i x u} d \mathrm{X}(u)-\lambda(x) \int_{-S_{2}}^{S_{2}} \int_{-S_{1}}^{S_{1}} e^{i x u} d \phi(u)
$$

is restrictedly convergent to zero as $S_{1}, S_{2} \rightarrow \infty$, uniformly in $x$.

The proof that the product is defined is similar to that for the circular case, except that now the set $(-u ; A)$ is contained in a set $[r+a, r+a]$ $\Theta[r, r]$, and condition $\mathrm{W}_{0}^{*}$ is used instead of (18). Proceeding as in Theorem 1 , we suppose that $x=0$ and write (46) as 


$$
\Delta_{S}=\int_{-\infty}^{+\infty} \int_{-\infty}^{+\infty}\{\phi(-u ;[S])-\phi([S])\} d \psi(u)
$$

Again, in view of the hypothesis on $\psi$ it is clear that the theorem will be proved if we can demonstrate the validity of the following statement. Given any pair of fixed positive real numbers $A_{1}$ and $A_{2}$, then for any $\epsilon>0$

$$
|\phi(-u ;[S])-\phi([S])| \leqq \epsilon\left(\left|u_{1}\right|+1\right)\left(\left|u_{2}\right|+1\right)
$$

provided that $A_{1} \leqq S_{1} / S_{2} \leqq A_{2}$ and $S_{1}>S_{0}, S_{2}>T_{0}, T_{0}$ and $S_{0}$ being fixed but dependent on $\epsilon$.

First let us suppose that $u$ does not lie in the interior of $\left[2 S_{1}, 2 S_{2}\right]$. Thus $\left|u_{1}\right| \geqq 2 S_{1}$ or $\left|u_{2}\right| \geqq 2 S_{2}$. To simplify our notation we assume that $-u$ is in the first quadrant. Then the set $(-u ;[S])$, which is the rectangle with center at $-u$ and sides of length $2 S_{1}$ and $2 S_{2}$, is contained in the square $[\|-u+S\|$, $\|-u+S\|]$. Hence

$$
\begin{aligned}
|\phi(-u ;[S])-\phi([S])| & \leqq|\phi(-u ;[S])|+|\phi([S])| \\
& \leqq W(\phi ;(-u ;[S]))+W(\phi ;[S])
\end{aligned}
$$

and $W(\phi ;(-u ;[S]))=o(\|-u+S\|)$. For the sake of definiteness let us assume now that $\|-u+S \mid=\left(\left|u_{1}\right|+S_{1}\right)$. If $\left|u_{1}\right| \geqq 2 S_{1}$, then $\left(\left|u_{1}\right|+S_{1}\right)$ is clearly majorized by $2\left(\left|u_{1}\right|+1\right)$. On the other hand, if $\left|u_{1}\right|<2 S_{1}$, we must have $\left|u_{2}\right| \geqq 2 S_{2}$. But since $A_{1} \leqq S_{1} / S_{2} \leqq A_{2}$, we see that there exists a constant $K$ such that $S_{1} \leqq K S_{2}$ and $S_{2} \leqq K S_{1}$. Therefore, $\left(\left|u_{1}\right|+S_{1}\right) \leqq\left(\left|u_{1}\right|+K S_{2}\right)$ $\leqq\left(\left|u_{1}\right|+1\right)+K\left(\left|u_{2}\right|+1\right)=O\left(\left(\left|u_{1}\right|+1\right)\left(\left|u_{2}\right|+1\right)\right)$. In summary, we have shown that

$$
W(\phi ;(-u ;[S]))=o\left(\left(\left|u_{1}\right|+1\right)\left(\left|u_{2}\right|+1\right)\right) .
$$

It is quite apparent that with appropriate modifications of sign the reasoning above is valid for any $u$. We next show that

$$
W(\phi ;[S])=o\left(\left(\left|u_{1}\right|+1\right)\left(\left|u_{2}\right|+1\right)\right) .
$$

Clearly, $[S] \subset[\|S\|,\|S\|]$ and so $W(\phi ;[S])=o(\|S\|)$. We have already seen that $o(\|S\|)=o\left(S_{1}\right)=o\left(S_{2}\right)$, and since either $\left|u_{1}\right|>2 S_{1}$ or $\left|u_{2}\right|>2 S_{2}$, the relation (50) holds. Combining (48), (49), and (50) shows that the right-hand side of $(48)$ is $o\left(\left(\left|u_{1}\right|+1\right)\left(\left|u_{2}\right|+1\right)\right)$.

Suppose now that $u$ lies in the interior of $[2 S] \ominus[S / 2]$. Then

$$
|\phi(-u ;[S])-\phi([S])| \leqq W(\phi ;[3 S])+W(\phi ;[S]) .
$$

An analysis similar to that used to establish (50) will show that each term on the right is $o\left(\left(\left|u_{1}\right|+1\right)\left(\left|u_{2}\right|+1\right)\right)$.

Lastly we consider the case in which $u$ lies in $[S / 2]$. In order to avoid complicating our notations and descriptions any more than is necessary, we shall suppose that $S_{1}=\|S\|$ and that $-u$ lies in the first quadrant. From the 
proof for this special situation it will be clear that the result we obtain is valid in general. We write

Now:

$$
\begin{aligned}
|\phi(-u ;[S])-\phi([S])| \leqq & W\left(\phi ;\left[\left|u_{1}\right|+S_{1},\left|u_{2}\right|+S_{2}\right] \ominus\left[S_{1}, S_{2}\right]\right) \\
& +W\left(\phi ;\left[S_{1}, S_{2}\right] \ominus\left[S_{1}-\left|u_{1}\right|, S_{2}-\left|u_{2}\right|\right]\right. \\
= & C+D .
\end{aligned}
$$

$$
\begin{aligned}
D \leqq & W\left(\phi ;\left[S_{1}-\left|u_{1}\right|+\left|u_{2}\right|, S_{2}\right] \ominus\left[S_{1}-\left|u_{1}\right|, S_{2}-\left|u_{2}\right|\right]\right) \\
& +W\left(\phi ;\left[S_{1}, S_{1}\right] \ominus\left[S_{1}-\left|u_{1}\right|+\left|u_{2}\right|, S_{1}-\left|u_{1}\right|+\left|u_{2}\right|\right]\right) \\
= & D_{1}+D_{2} .
\end{aligned}
$$

Note that whenever $\left|u_{2}\right| \geqq\left|u_{1}\right|,\left[S_{1}, S_{1}\right] \ominus\left[S_{1}-\left|u_{1}\right|+\left|u_{2}\right|, S_{1}-\left|u_{1}\right|+\left|u_{2}\right|\right]$ is the null set. Thus since $\phi$ satisfies $\mathrm{W}_{0}^{*}$,

$$
\begin{aligned}
& D_{1}=o\left(\left|u_{2}\right|\right) \leqq o\left(\left(\left|u_{1}\right|+1\right)\left(\left|u_{2}\right|+1\right)\right), \\
& D_{2}=o\left(\left|u_{1}\right|-\left|u_{2}\right|\right) \leqq o\left(\left(\left|u_{1}\right|+1\right)\left(\left|u_{2}\right|+1\right)\right) .
\end{aligned}
$$

Assuming that $\left|u_{1}\right| \geqq\left|u_{2}\right|$, we have

$$
\begin{aligned}
C & \leqq W\left(\phi ;\left[\left|u_{1}\right|+S_{1},\left|u_{1}\right|+S_{2}\right] \ominus\left[S_{1}, S_{2}\right]\right) \\
& =o\left(\left|u_{1}\right|\right) \leqq o\left(\left(\left|u_{1}\right|+1\right)\left(\left|u_{2}\right|+1\right)\right) .
\end{aligned}
$$

Similarly if $\left|u_{2}\right|>\left|u_{1}\right|$, we get that $C=o\left(\left(\left|u_{1}\right|+1\right)\left(\left|u_{2}\right|+1\right)\right)$. This completes the proof of the theorem under the assumption that $x=0$. For the case of general $x$ we proceed as we did in the circular case, making necessary changes to fit the case at hand. With the details for $x=0$ given above, the reader should have no trouble filling in the proof.

Lemma 6, which follows, gives Leibniz's rule for differentiating the products of integrals which obey certain conditions. Its proof is patterned along that of Lemma 5 and is omitted.

Lemma 6. Let $B$ and $G$ be two trigonometric integrals as in (7). Further, let $\iint_{A(b)}\left|u_{1}\right| K\left|u_{2}\right| L|d \phi(u)|=o(1)$ rapidly as $b_{1}, b_{2} \rightarrow \infty$ restrictedly, and let $\iint_{E_{2}}\left(\left|u_{1}\right|+1\right)^{K}\left(\left|u_{2}\right|+1\right)^{L}|d \psi(u)|<\infty$. Then the formal product $P=B G$ exists, and

$$
\frac{\partial^{K+L}}{\partial x_{1}^{K} \partial x_{2}^{L}} P=(i)^{K+L} \sum_{k, l=0}^{K, L}\left(\begin{array}{c}
K \\
k
\end{array}\right)\left(\begin{array}{c}
L \\
l
\end{array}\right)\left\{\frac{\partial^{K-k+L-l}}{\partial x_{1}^{K-k} \partial x_{2}^{L-l}} B\right\}\left\{\frac{\partial^{k+l}}{\partial x_{1}^{k} \partial x^{l}} G\right\}
$$

where all differentiations and products are formal.

3.2. Localization and equiconvergence. As in the circular case, basic facts about Fourier integrals and the notions of localizing function and Riemann function are indispensable. We list briefly some of the modifications of these ideas which are made in the restricted case. 
Instead of considering the circular partial Fourier integral we now consider the ordinary rectangular partial integral. That is, if $F(t)$ is integrable and $\gamma(u)$ is its Fourier transform, we consider

$$
\int_{-S_{2}}^{S_{2}} \int_{-S_{1}}^{S_{1}} \gamma(u) e^{i x u} d u=\frac{1}{\pi^{2}} \int_{-\infty}^{+\infty} \int_{-\infty}^{+\infty} F(t) \bar{D}_{S}(x-t) d t,
$$

where

$$
\bar{D}_{S}(x) \equiv \bar{D}_{S_{1}}\left(x_{1}\right) \bar{D}_{S_{2}}\left(x_{2}\right) ; \quad \bar{D}_{S_{i}\left(x_{i}\right)}=\frac{\sin S_{i} x_{i}}{x_{i}}, \quad i=1,2 .
$$

In the consideration of localizing functions we specialize our domains to be rectangles and assume that the localizing functions we use are the products of the one-dimensional localizing functions associated with the projections of the rectangles onto the coordinate axes. Explicit constructions of one-dimensional localizing functions are given in [4].

Let $B$ be a trigonometric integral as in (7), where $\phi$ satisfies condition $W_{0}$. The Riemann function $F(x)$ associated with $B$ is defined by formal integration as follows:

$$
\begin{aligned}
F(x)= & \int_{-1}^{1} \int_{-1}^{1}\left\{\frac{e^{i x_{1} u_{1}}-1-i x_{1} u_{1}}{\left(i u_{1}\right)^{2}}\right\}\left\{\frac{e^{i x_{2} u_{2}}-1-i x_{2} u_{2}}{\left(i u_{2}\right)^{2}}\right\} d \phi(u) \\
& +\int_{-1}^{1}\left(\int_{-\infty}^{-1}+\int_{1}^{\infty}\right) \frac{e^{i x_{1} u_{1}}}{\left(i u_{1}\right)^{2}}\left\{\frac{e^{i x_{2} u_{2}}-1-i x_{2} u_{2}}{\left(i u_{2}\right)^{2}}\right\} d \phi(u) \\
& +\left(\int_{-\infty}^{-1}+\int_{1}^{\infty}\right) \int_{-1}^{1}\left\{\frac{e^{i x_{1} u_{1}}-1-i x_{1} u_{1}}{\left(i u_{1}\right)^{2}}\right\} \frac{e^{i x_{2} u_{2}}}{\left(i u_{2}\right)^{2}} d \phi(u) \\
& +\iint_{\Gamma} \frac{e^{i x u} d \phi(u)}{\left(i u_{1}\right)^{2}\left(i u_{2}\right)^{2}}=F_{1}(x)+F_{2}(x)+F_{3}(x)+F_{4}(x),
\end{aligned}
$$

where $\Gamma$ denotes integration over that region not covered by the first three integrals. Clearly, the integrals all converge uniformly in $x$.

Theorem 7. Let $B=\int_{-\infty}^{+\infty} \int_{-\infty}^{+\infty} e^{i x u} d \phi(u)$ where $\phi$ satisfies the condition $\mathrm{W}_{0}$. Suppose further that $\Re$ and $\Re_{1}$ are two closed bounded rectangles, $\Re \subset \Re_{1}^{\circ}$, and that $\lambda(x)$ is a localizing function of class $C^{(10)}$ associated with $\Re$ and $\Re_{1}$. Then if $F(x)$ is the Riemann function associated with $B$, the difference

$$
\begin{aligned}
\Delta_{S_{1} S_{2}}= & \int_{-S_{2}}^{S_{2}} \int_{-S_{1}}^{S_{1}} e^{i x u} d \phi(u) \\
& -\frac{1}{\pi^{2}} \int_{-\infty}^{+\infty} \int_{-\infty}^{+\infty} F(t) \lambda(t) \frac{\partial^{2} \bar{D}_{S_{1}}\left(x_{1}-t_{1}\right)}{\partial t_{1}^{2}} \frac{\partial^{2} \bar{D}_{S_{2}}\left(x_{2}-t_{2}\right)}{\partial t_{2}^{2}} d t_{1} d t_{2}
\end{aligned}
$$

is restrictedly convergent to zero as $S_{1}, S_{2} \rightarrow \infty$, uniformly for $x$ in $\Re$. 
Step one in the proof is to write $\phi$ as the sum of four set functions $\phi_{1}, \phi_{2}, \phi_{3}, \phi_{4}$, defined as follows: $\phi_{1}(E)=\phi([1,1] \wedge E) ; \phi_{2}(E)=\phi(\mathfrak{X} \wedge E)$, where $\mathfrak{X}=\left\{\left(x_{1}, x_{2}\right) ;\left|x_{1}\right|>1,\left|x_{2}\right| \leqq 1\right\} ; \phi_{3}(E)=\phi(\mathfrak{Y} \wedge E)$, where $\mathfrak{V}=\left\{\left(x_{1}, x_{2}\right)\right.$; $\left.\left|x_{2}\right|>1,\left|x_{1}\right| \leqq 1\right\}$; and $\phi_{4}(E)=\phi(ß \wedge E)$, where $\nexists$ is the complement of $[1,1]+\mathfrak{X}+\mathfrak{Y}$. Clearly it suffices to prove the theorem for each of the functions $\phi_{1}$ to $\phi_{4}$ separately.

The case of $\phi_{1}$ corresponds to the case $\phi(E)=0$ for $E$ exterior to $C_{1}$ in the discussion of circular convergence. The Riemann function is $F_{1}(x)$, which is regular in $x_{1}$ and $x_{2}$. Since there is no essential difference in the proofs between the restricted case and the corresponding circular case, we omit the proof. Also, the case of $\phi_{4}$ corresponds to the assumption that $W\left(\phi ; C_{1}\right)=0$ in the circular situation. Again, the proof of the present theorem for $\phi_{4}$ does not differ much from the proof in the circular case when we assume $W\left(\phi ; C_{1}\right)$ $=0$. Our Riemann function now is given by $F_{4}(x)$, the function $\Phi$ used in the proof will now be defined as $\Phi(E)=\iint_{E} d \phi(u) /\left(i u_{1}\right)^{2}\left(i u_{2}\right)^{2}$ and $\mu(x)$ will satisfy the order condition $o\left(\left(\left|x_{1}\right|+1\right)^{-5 / 2}\left(\left|x_{2}\right|+1\right)^{-5 / 2}\right)$.

The analysis which deals with the functions $\phi_{2}$ and $\phi_{3}$ does present some novel features. We shall consider $\phi_{2}$ and henceforth drop the subscript. To make matters definite we take the rectangle $\Re$ to be the cartesian product of the intervals $a_{1} \leqq x_{1} \leqq b_{1}$ and $a_{2} \leqq x_{2} \leqq b_{2}$, and take $\Re_{1}$ to be the product of $c_{1} \leqq x_{1} \leqq d_{1}$ and $c_{2} \leqq x_{2} \leqq d_{2} ; c_{1}<a_{1}<b_{1}<d_{1}, c_{2}<a_{2}<b_{2}<d_{2}$. If we denote the localizing function associated with the projections on the $x_{1}$-axis and the $x_{2}$-axis by $\mu\left(x_{1}\right)$ and $\nu\left(x_{2}\right)$ respectively, we have $\lambda\left(x_{1}, x_{2}\right)=\mu\left(x_{1}\right) \nu\left(x_{2}\right)$.

First observe that $F_{2}(x) \equiv F(x)=F\left(x_{1}, x_{2}\right)$ is continuous in both variables and is regular in $x_{2}$. Let us next define a function on the Borel sets of the $x_{1-}$ axis as follows:

$$
\phi_{x}^{\prime}(A)=\iint_{I \times A} e^{i x_{2} u_{2}} d \phi(u)
$$

where $I \times A$ is the cartesian product of the interval $-1 \leqq x_{2} \leqq 1$ and the onedimensional Borel set $A$. One sees immediately that $\phi_{x}^{\prime}$ depends on $x_{2}$ and for fixed $x_{2}$ satisfies the conditions 1 (a) and 1 (b) applied to one-dimensional Borel sets. Furthermore,

$$
\int_{v}^{v+1}\left|d \phi_{x}^{\prime}\left(u_{1}\right)\right|=o\left(|v|^{-1 / 2}\right), \quad \text { uniformly in } x,
$$

and

$$
\int_{-1}^{1} \int_{a}^{b} e^{i x u} d \phi(u)=\int_{a}^{b} e^{i x_{1} u_{1}} d \phi_{x}^{\prime}\left(u_{1}\right)
$$

Relation (54) follows from the fact that $\phi$ is in $W_{0}^{*}$. Equation (55) can be established by writing the right-hand side as an iterated limit and noting that 
the iterated limit is equal to a double limit which can be used to define the left-hand member. Our purpose in introducing (55) is to enable us to consider $\int_{-1}^{1} \int_{-\infty}^{+\infty} e^{i x u} d \phi(u)$ as the one-dimensional integral $\int_{-\infty}^{+\infty} e^{i x_{1} u_{1}} d \phi_{x}^{\prime}\left(u_{1}\right)$ and to apply the one-dimensional theory developed in [4] to the latter integral. From the results in [4], from (55), and the definition of $F$, one readily sees that for $a_{2} \leqq x_{2} \leqq b_{2}$ the one-dimensional Riemann function associated with $\int_{-\infty}^{+\infty} e^{i x_{1} u_{1}} d \phi_{x}^{\prime}(u)$ is

$$
\frac{\partial^{2}}{\partial x_{2}^{2}}\left\{F\left(x_{1}, x_{2}\right) \nu\left(x_{2}\right)\right\}=G\left(x_{1}, x_{2}\right) .
$$

Again applying known results in one dimension [5], we see that

$$
\int_{-1}^{1} \int_{-S_{1}}^{S_{1}} e^{i x u} d \phi(u)-\frac{1}{\pi} \int_{-\infty}^{+\infty} G\left(s_{1}, x_{2}\right) \mu\left(s_{1}\right) \frac{\partial^{2}}{\partial s_{1}^{2}} \bar{D}_{S_{1}}\left(x_{1}-s_{1}\right) d s_{1}
$$

tends to zero as $S_{1} \rightarrow \infty$, uniformly for $x$ in $\Re$. Since $\nu\left(x_{2}\right)$ has six continuous derivatives, it is clear from (56) that $G\left(s_{1}, x_{2}\right)$ has four continuous derivatives with respect to $x_{2}$ which are bounded for all values of $\left(s_{1}, x_{2}\right)$ and that $G\left(s_{1}, x_{2}\right)$ is integrable over $(-\infty,+\infty)$ as a function of $x_{2}$. Thus we have

$$
G\left(s_{1}, x_{2}\right)=\int_{-S_{2}}^{S_{2}} e^{i x_{2} t} \theta\left(s_{1}, t\right) d t+\left(\int_{-\infty}^{-S_{2}}+\int_{S_{2}}^{+\infty}\right) e^{i x_{2} t \theta\left(s_{1}, t\right) d t}
$$

where

$$
\theta\left(s_{1}, t\right)=\frac{1}{2 \pi} \int_{-\infty}^{+\infty} G\left(s_{1}, x_{2}\right) e^{-i x_{2} t} d x_{2}=o\left(|t|^{-4}\right) \quad \text { as }|t| \rightarrow \infty,
$$

the small " $o$ " holding uniformly in $s_{1}$. Furthermore, since $\lambda\left(x_{1}, x_{2}\right)=\mu\left(x_{1}\right) \nu\left(x_{2}\right)$ we have $\partial^{2} F\left(x_{1}, x_{2}\right) \lambda\left(x_{1}, x_{2}\right) / \partial x_{2}^{2}=G\left(x_{1}, x_{2}\right) \mu\left(x_{1}\right)$ and can now rewrite the righthand side of (53) as

$$
\begin{aligned}
& \Delta_{S_{1}, S_{2}}=\Delta_{S_{1}, S_{2}}^{\prime}+\Delta_{S_{1}, S_{2}}^{\prime \prime} \\
& =\left\{\int_{-1}^{1} \int_{-S_{1}}^{S_{1}} e^{i x u} d \phi(u)\right. \\
& -\frac{1}{\pi^{2}} \int_{-\infty}^{+\infty}\left(\int_{-\infty}^{+\infty} G\left(s_{1}, s_{2}\right) \bar{D}_{S_{2}}\left(x_{2}-s_{2}\right) d s_{2}\right) \mu\left(s_{1}\right) \frac{\partial^{2} \bar{D}\left(x_{1}-s_{1}\right)}{\partial s_{1}^{2}} d s_{1}
\end{aligned}
$$

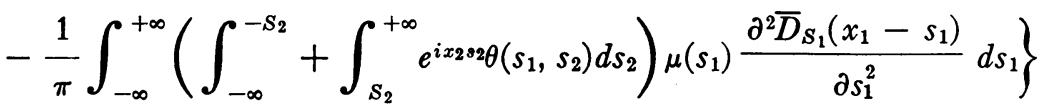

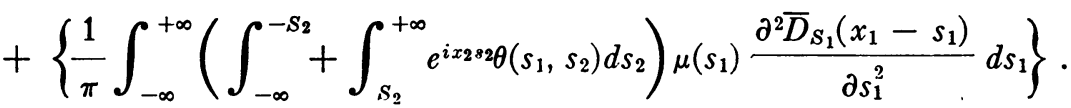

From (58) we see that $\Delta_{s_{1}, s_{2}}^{\prime}$ is merely the difference (57), and so 


$$
\lim _{S_{1}, S_{2} \rightarrow \infty} \Delta_{S_{1}, S_{2}}^{\prime}=\lim _{S_{1} \rightarrow \infty} \Delta_{S_{1}, S_{2}}^{\prime}=0 .
$$

Using the estimate in (59), the bound $\partial^{2} \bar{D}_{S_{1}}\left(x_{1}-s_{1}\right) / \partial s_{1}^{2}=O\left(\left|S_{1}\right|^{3}\right)$, the fact that the ratio $S_{1} / S_{2}$ is bounded, and the fact that $\mu\left(s_{1}\right)=0$ outside of a finite interval, we immediately see that $\Delta_{s_{1}, s_{2}}^{\prime \prime} \rightarrow 0$ restrictedly and thereby complete the proof.

Corresponding to Theorem 3 we now have the following situation.

THEOREM 8. Let $B=\int_{-\infty}^{+\infty} \int_{-\infty}^{+\infty} e^{i x u} d \phi(u)$ be a double trigonometric integral such that $\phi$ satisfies the condition $\mathrm{W}_{0}^{*}$. If $\Re^{*}$ is a closed bounded rectangle contained in the interior of $\Omega$, then there is a double trigonometric series $\sum a_{m} e^{i m x}$ whose coefficients satisfy the condition $a_{m}=o\left(\left(\left|m_{1}\right|+1\right)^{-1 / 2}\left(\left|m_{2}\right|+1\right)^{-1 / 2}\right)$ rapidly and such that

$$
\int_{-S_{2}}^{S_{2}} \int_{-S_{1}}^{S_{1}} e^{i x u} d \phi(u)-\sum_{\left|m_{1}\right|,\left|m_{2}\right|=0}^{\left[S_{1}\right],\left[S_{2}\right]} a_{m} e^{i m x}
$$

converges restrictedly to zero as $S_{1}, S_{2} \rightarrow \infty$, uniformly in $x$.

As in the proof of the preceding theorem, we write $\phi=\phi_{1}+\phi_{2}+\phi_{3}+\phi_{4}$. For functions $\phi_{1}$ and $\phi_{4}$ the proof of Theorem 8 is, except for minor modifications, the same as that of Theorem 3. To treat $\phi_{2}$ we first transform the integral $\int_{-1}^{1} \int_{-\infty}^{+\infty} e^{i x u} d \phi_{2}(u)$ into an integral $\int_{-\infty}^{+\infty} e^{i x_{1} u} 1 \phi_{z}^{\prime}\left(u_{1}\right)$ with Riemann function $G\left(x_{1}, x_{2}\right)$ as was done in the preceding theorem. Using one-dimensional results of $Z$ ygmund [5] we show that our integral is equiconvergent with a series

$$
\sum_{m_{1}=-\infty}^{+\infty} a_{m_{1}}\left(x_{2}\right) e^{i m_{1} x_{1}} ; \quad a_{m_{1}}\left(x_{2}\right)=o\left(\left(\left|m_{1}\right|+1\right)^{-1 / 2}\right) .
$$

This series is obtained by expanding the function $\Gamma\left(x_{1}, x_{2}\right)$, which is of period $2 \pi$ and equals $G\left(x_{1}, x_{2}\right) \mu\left(x_{1}\right)$ on $\Omega$, in a Fourier series in $x_{1}$ and then formally differentiating the series twice with respect to $x_{1}$. The coefficients $a_{m_{1}}\left(x_{2}\right)$ will be periodic functions of $x_{2}$ and can be taken to have as many derivatives as we please. Thus the functions $a_{m}\left(x_{2}\right)$ can be expanded in Fourier series with coefficients $a_{m_{1} m_{2}}=o\left(\left|m_{2}\right|^{-3}\right)$, uniformly in $m_{1}$. Hence

$$
\sum_{-M_{1}}^{M_{1}} a_{m_{1}}\left(x_{2}\right) e^{i m_{1} x_{1}}=\sum_{-M_{1},-M_{2}}^{M_{1}, M_{2}} a_{m_{1} m_{2}} e^{i\left(m_{1} x_{1}+m_{2} x_{2}\right)}+\sum_{-M_{1}}^{M_{1}} o\left(\frac{1}{M_{2}^{2}}\right) e^{i m_{1} x_{1}} .
$$

From this it follows that, since $M_{1} / M_{2}$ is bounded,

$$
\left\{\int_{-1}^{1} \int_{-M_{1}}^{M_{1}} e^{i x u} d \phi_{3}(u)-\sum_{-M_{1},-M_{2}}^{M_{1}, M_{2}} a_{m_{1} m_{2}} e^{i\left(m_{1} x_{1}+m_{2} x_{2}\right)}\right\} \rightarrow 0
$$

restrictedly, as $M_{1}, M_{2} \rightarrow \infty$, uniformly for $x$ in $\Re^{*}$.

Finally, we observe that the analogue of Theorem 4 and the remarks con- 
cerning the conjugate integral are also true in the restricted case. We leave their explicit formulation and proof to the reader.

\section{REFERENCES}

1. L. D. Berkovitz, Circular summation and localization of double trigonometric series, Trans. Amer. Math. Soc. vol. 70 (1951) pp. 323-344.

2. S. Saks, Theory of the integral, Warsaw, 1937.

3. František Wolf, Contributions to a theory of summability of trigonometric integrals, University of California Publications in Mathematics, New Series, vol. 1, pp. 159-228.

4. A. Zygmund, Sur la théorie riemannienne des séries trigonometriques, Math. Zeit. vol. 24 (1926) pp. 47-104.

5. - On trigonometric integrals, Ann. of Math. vol. 48 (1947) pp. 393-440.

University of Chicago,

Chicago, Ill. 